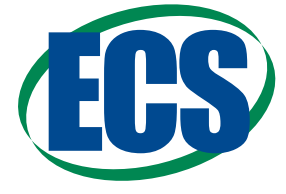

Jes Focus Issue on Advances in Electrochemical Processes for Interconnect Fabrication in Integrated Circuits

\title{
Review-Laser Ablation Ionization Mass Spectrometry (LIMS) for Analysis of Electrodeposited Cu Interconnects
}

\author{
Valentine Grimaudo, ${ }^{1}{ }^{1}$ Pavel Moreno-García, ${ }^{1}{ }^{1}$ Andreas Riedo, ${ }^{2}{ }^{2,3}$ \\ Alena Cedeño López, $\oplus^{1}$ Marek Tulej, $\oplus^{3}$ Reto Wiesendanger, $\oplus^{3}$ Peter Wurz, $\oplus^{3}$ \\ and Peter Broekmann ${ }^{1, *, z}$ \\ I Department of Chemistry and Biochemistry, Interfacial Electrochemistry Group, 3012 Bern, Switzerland
${ }^{2}$ Sackler Laboratory for Astrophysics, Leiden Observatory, Leiden University, 2333 CA Leiden, The Netherlands
${ }^{3}$ Physics Institute, Space Research and Planetary Sciences, University of Bern, 3012 Bern, Switzerland
}

\begin{abstract}
In this contribution highly sensitive and quantitative analytical methodologies based on femtosecond Laser Ablation Ionization Mass Spectrometry (fs-LIMS) for the analysis of model systems and state-of-the-art $\mathrm{Cu}$ interconnects are reviewed and discussed. The method development introduces in a first stage a 1D chemical depth profiling approach on electrodeposited $\mathrm{Cu}$ films containing periodically confined organic layers. Optimization of measurement conditions on these test platforms enabled depth profiling investigations with vertical resolution at the $\mathrm{nm}$ level. In a second stage, a matrix-free laser desorption methodology was developed that allowed for preliminary molecular identification of the embedded organic contaminants beyond elementary composition. These studies provided specific fragmentation markers in the lower mass range, which support a previously proposed reaction mechanism responsible for successful leveling employing a new class of plating additives for Damascene processes. Further combined LIMS and Scanning Auger Microscopy (SAM) studies on through-silicon-vias (TSV) interconnects confirmed the embedment upon plating of the organic additives at the upper side-walls of the TSV channel in the boundary between the $\mathrm{Cu}$ seed layer and the electrodeposited $\mathrm{Cu}$.

(C) The Author(s) 2018. Published by ECS. This is an open access article distributed under the terms of the Creative Commons Attribution 4.0 License (CC BY, http://creativecommons.org/licenses/by/4.0/), which permits unrestricted reuse of the work in any medium, provided the original work is properly cited. [DOI: 10.1149/2.0221901jes]

(cc) BY
\end{abstract}

Manuscript submitted September 25, 2018; revised manuscript received November 14, 2018. Published December 1, 2018. This paper is part of the JES Focus Issue on Advances in Electrochemical Processes for Interconnect Fabrication in Integrated Circuits.

The $\mathrm{Cu}$ interconnect technology of today's cutting-edge microprocessors relies, to a large extent, on additive-assisted electroplating processes, which allow for fast, cost-effective and void-free bottomup filling of vias and trenches. ${ }^{1-7}$ Incorporation of minor and even trace amounts of the employed organic additives in the deposited material as a result of the plating process is, however, an important drawback that might undermine the reliability, performance and lifespan of the manufactured interconnects. ${ }^{8-11}$ Hence new plating formulations with functionalities beyond the standard concept of Damascene processing that prevent or minimize such additive embedment have been designed and implemented. ${ }^{12-15}$ In this context, accurate and comprehensive characterization of the interconnect features manufactured with these improved plating additive packages requires their quantitative chemical analysis with a spatial resolution at micro- and nanometer level. To date a variety of analytical techniques are available that can be applied for the chemical analysis of integrated circuits (IC). Based on their particular principles and figures of merit, they have proven useful for characterization and quality control of many solid materials relevant for the semiconductor industry. According to their specific spatial analysis capabilities, these techniques can be grouped in surface sensitive techniques, e.g. X-ray Photoelectron Spectroscopy (XPS), ${ }^{16,17}$ Auger Electron Spectroscopy (AES) ${ }^{16,18}$ and (Nano)-Secondary Ion Mass Spectrometry (Nano-SIMS), ${ }^{17-20}$ and bulk-probing methods, e.g. Laser Ablation Inductively Coupled Plasma Mass Spectrometry (LA-ICP-MS), ${ }^{21-24}$ Laser-Induced Breakdown Spectroscopy (LIBS), ${ }^{24,25}$ and Glow Discharge Mass Spectrometry (GD-MS). ${ }^{24,26}$

In this framework, Laser Ablation Ionization Mass Spectrometry (LIMS) currently emerges as an innovative experimental approach that, contrary to some of the above-mentioned techniques, does not require any sample preparation step, exhibits minimized matrix effects and enables therefore standardless experimental procedures. Additionally, it offers high detection sensitivity coupled to high vertical and lateral spatial resolution.

\footnotetext{
*Electrochemical Society Member.

${ }^{\mathrm{z} E}$-mail: peter.broekmann@dcb.unibe.ch
}

The first LIMS system, LAMMA-500, was introduced by Hillenkamp et al. in the mid-seventies. ${ }^{27}$ Subsequently, various LIMS prototypes with different instrument designs (e.g., coaxial ${ }^{28-34}$ or offaxis $^{35-37}$ geometry of the analyzer with respect to the incident laser beam) have been constructed for applications ranging from imaging of biological tissues ${ }^{38}$ to the identification of impurities in dielectrics of microelectronics. ${ }^{39}$ Continuous advances in electronics and vacuum technology, ${ }^{29}$ laser systems ${ }^{40-42}$ in conjunction with the development of new ion trajectory simulation software packages (e.g. SIMION) have made this technique to re-emerge for laboratory and field applications.

This contribution is intended to review the major recent advances developed for a home-made LIMS system (Laser Mass Spectrometer (LMS), see Fig. 1) 28-30,43,44 $^{2}$ whose chemical analysis capabilities were applied on electrochemically manufactured testbeds relevant for state-of-the-art interconnect technology. Emphasis was placed on the analysis of electroplated $\mathrm{Cu}$ films that were prepared using a new Damascene plating concept based on bis-sodium-sulfopropyldisulfide (SPS) $)^{1,45}$ and a co-polymer of imidazole and epichlorohydrin (IMEP) $)^{12-15,20}$ organic additives. Particular attention was devoted to address the specific interaction between SPS and IMEP under reactive plating conditions, which supports the validity of a deposition reaction mechanism recently proposed (Fig. 2). ${ }^{12-14,20}$ While IMEP is a well-known leveler, it has been also demonstrated that in combination with SPS it exhibits, under specific experimental conditions, a suppressor-leveler bi-functionality. It could therefore replace commonly applied suppressor additives,( e.g. PEG), enabling a simplified plating chemistry for the superconformal $\mathrm{Cu}$ growth of Damascene structures. In a further stage, actual TSVs interconnects manufactured with analogue plating chemistry were scrutinized and their spatial chemical constitution addressed by the proposed LIMS approach.

\section{Experimental}

Electrochemical deposition of Cu testbeds.-Electrochemical deposition of the test platforms were carried out under galvanostatic conditions (constant applied current density $j\left[\mathrm{mAcm}^{-2}\right]$ ) with a three electrode electrochemical cell connected to an Autolab 

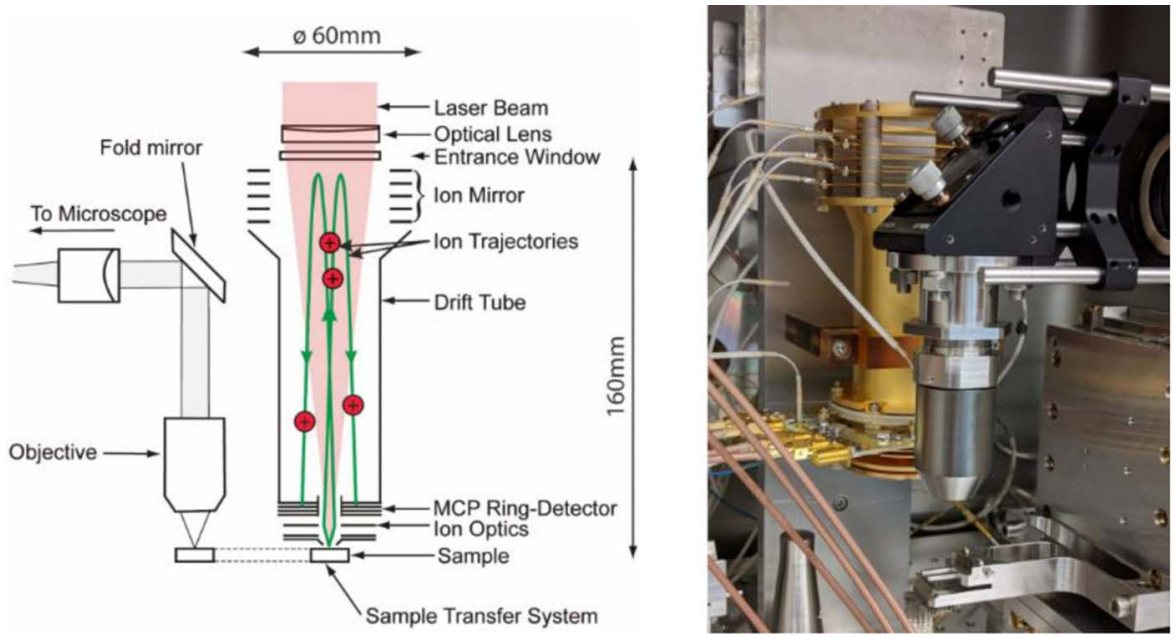

Figure 1. Illustration of the LMS system coupled to the UHV-compatible microscope system. ${ }^{52}$

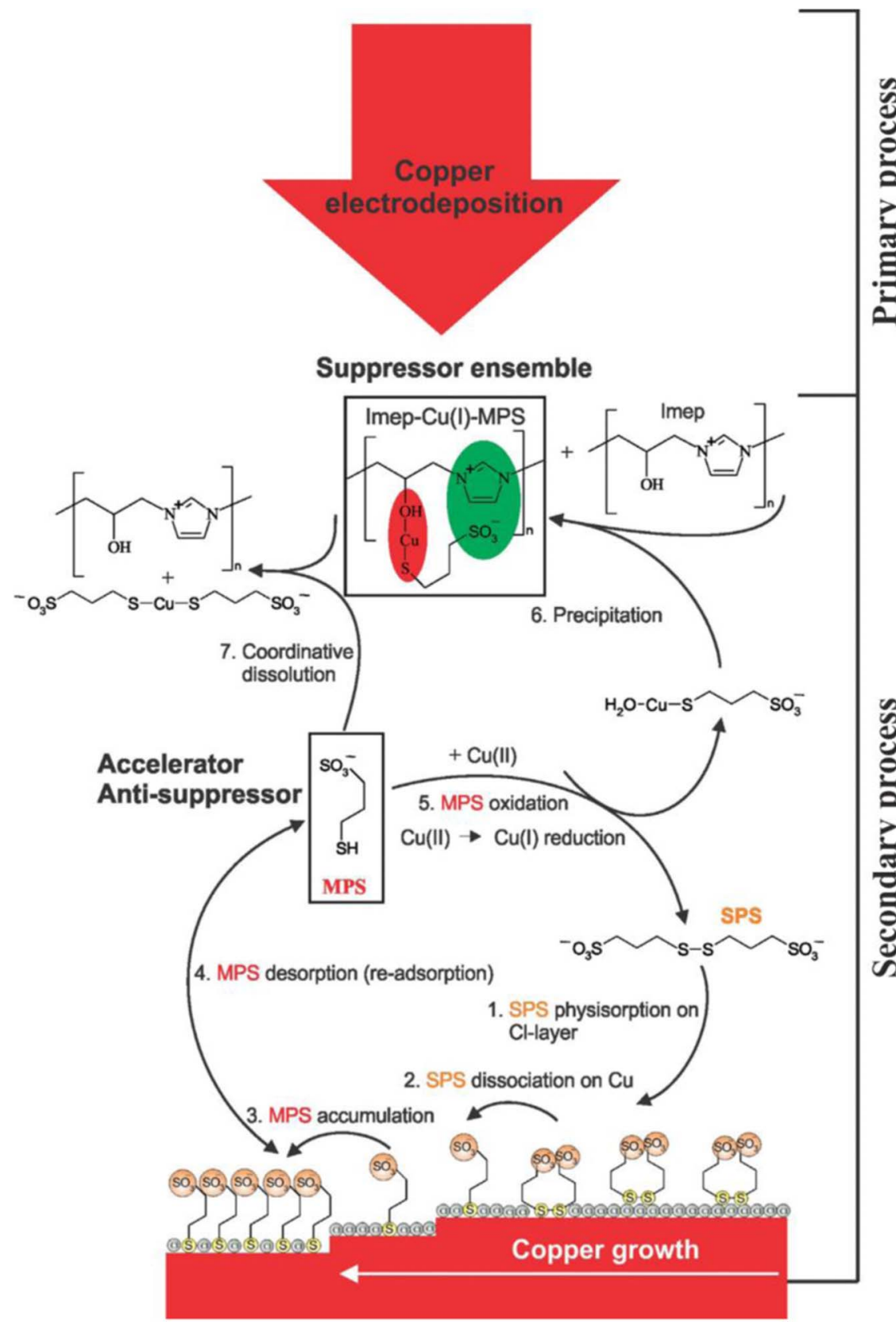

Figure 2. Proposed reaction scheme for the MPS/IMEP interaction during reactive deposition conditions. Scheme reprinted with permission from Ref. 12. Copyright (2012) Elsevier. 
Table I. Plating conditions applied to prepare the various electrochemical deposits analyzed by LIMS.

\begin{tabular}{|c|c|c|c|}
\hline Experiment & Plating package & Nominal current density & Thickness of the deposit \\
\hline 1 & Blank deposit & $-10 \mathrm{mAcm}^{-2}$ & $10 \mu \mathrm{m}$ \\
\hline 2 & 25 ppm SPS, 100 ppm PAG & $-10 \mathrm{mAcm}^{-2}$ & $10 \mu \mathrm{m}$ \\
\hline 3 & 25 ppm SPS, 100 ppm PEI & $-10 \mathrm{mAcm}^{-2}$ & $10 \mu \mathrm{m}$ \\
\hline 4 & 25 ppm SPS, 100 ppm IMEP & $-4 \mathrm{mAcm}^{-2}$ to $-6 \mathrm{mAcm}^{-2}$ & $10 \mu \mathrm{m}$ to $15.4 \mu \mathrm{m}$ \\
\hline
\end{tabular}

potentiostat/galvanostat (PGSTAT 128). The cell consisted of a double junction $\mathrm{Ag} / \mathrm{AgCl}_{3 \mathrm{M}}$ reference electrode (Metrohm, Switzerland) and a Pt-wire counter electrode, which was placed inside a glass compartment holding a ceramic frit at the bottom. A commercial $\mathrm{Cu}$-seeded $\mathrm{Si}(100)$ wafer (Hionix, BASF, Ludwigshafen, Germany) served as working electrode. The aqueous electrolyte consisted of a copper sulfate solution $\left(40 \mathrm{~g} / \mathrm{L} \mathrm{Cu}^{2+}\left(\mathrm{CuSO}_{4} \cdot 5 \mathrm{H}_{2} \mathrm{O}\right)\right.$, Sigma-Aldrich, Steinheim, Germany) with a $\mathrm{H}_{2} \mathrm{SO}_{4}$ supporting electrolyte (10 g/L VLSI selectipur $96 \%$, BASF, Ludwigshafen, Germany) and $50 \mathrm{ppm} \mathrm{Cl}{ }^{-}$ ( $\mathrm{HCl} 25 \%$, for analysis, Merck, Darmstadt, Germany). Various plating additives were added to the electrolyte for the deposition of copper layers that exhibited distinct roughness characteristics, e.g. bis(sodium sulfopropyl) disulfide (SPS), polyalkylene glycol (PAG) (copolymer of polyethylene glycol and polypropylene glycol), polymerizate of imidazole and epichlorohydrin (IMEP), and polyethylenimine (PEI). SPS was acquired from Raschig (Ludwigshafen, Germany) and PAG, PEI and IMEP from BASF (Ludwigshafen, Germany), respectively. Table I summarizes the applied plating conditions for the preparation of the distinct samples.

Laser ablation ionization mass spectrometry (LIMS).Instrumentation.-The instrument consists of a miniature, gridless reflectron-type time-of-flight (RTOF) mass analyzer $(160 \mathrm{~mm} \times$ $\varnothing 60 \mathrm{~mm}$ ) and of a femtosecond-pulsed laser system for the ablation and ionization of solid material (Fig. 1). ${ }^{28-30,43,44,46-48}$ The TOF mass analyzer features several benefits over other mass spectrometric systems, among which are the high ion transmission that leads to very high sensitivity, and the capability to record all generated ions over a large mass range within a short timescale (in case of LMS only 20 microseconds for all elements and their isotopes). On the other hand, the ultrafast laser system enables fast and precise ablation and ionization of material from the surface ${ }^{39,48,49}$ with minimal thermal alteration of the irradiated zones, e.g. melting of the material. Laser ablation does not require any elaborated sample preparation step, which would alter and, more important, might introduce impurities to the sample. The large degree of freedom available for the adjustment of laser parameters, e.g. wave length, pulse width, pulse energy, repetition rate and number of applied laser pulses makes this technique sensitive to any kind of solid sample susceptible of absorbing laser light. Owing to its high detection sensitivity, the instrument requires only pico to femtogramms for comprehensive quantitative chemical analysis and is therefore considered to be a quasi-non-destructive measurement technique. ${ }^{30}$

The LMS is currently coupled to a Ti-Sapphire laser system, which generates at max. $1 \mathrm{kHz}$ repetition rate ultra-short Gaussian laser pulses with a pulse width of about $190 \mathrm{fs}$ at a wavelength of $775 \mathrm{~nm}$. The laser pulses are guided toward the mass analyzer via a set of dielectric mirrors and are focused by a lens system positioned on top of it to the sample surface along its ion optical axis and through an ion optical system at the detector unit (Fig. 1). The interaction of the laser beam with the sample surface at the focal point induces the ablation of the target material (Ø 10-20 $\mu \mathrm{m})$ and forms a hot plasma plume consisting of atomized and ionized species. To ensure that a major fraction of ionized species enters the mass analyzer (only positively charged species) the sample is positioned in close proximity to the skimmer, which is about $1 \mathrm{~mm}$ away, on a computer controlled $x-y-z$ translation stage. After having passed the second time the fieldfree region of the mass analyzer the ion packages arrive sequentially in time at the detector unit according to their mass to charge $\mathrm{m} / \mathrm{z}$ ratios, where they impact on two multi-channel plates (MCP, chevron configuration) and promote an electron avalanche that generates an electrical signal. A concentric anode system is integrated that collects the electrons and forwards them to two high-speed ADC cards. The current detector design allows to record spectra at a dynamic range of about eight orders of magnitude. ${ }^{50}$

The initialization of the laser triggers the data acquisition system to record a full TOF spectrum of $20 \mu \mathrm{s}$, which comprises a mass range of up to 400 mass/charge $(\mathrm{m} / \mathrm{z})$. Once a measurement is finished, the recorded time spectra are converted to mass spectra using the relation $m / z(t)=a\left(t-t_{0}\right)^{2}$, with $a$ and $t_{0}$ as instrument specific calibration factors that depend on the applied ion optical system. ${ }^{43}$ In-house designed software packages, written in Java/C $++/$ Matlab/Phyton are used for the sample positioning, laser system operation, data acquisition and analysis and processing of the acquired spectra. ${ }^{51}$

For the precise targeting of micrometer-sized features on the sample surface a microscope system with an imaging resolution of about $2 \mu \mathrm{m}$ is implemented in the vacuum chamber next to the mass analyzer (Fig. 1). ${ }^{52}$ The position of interest at the microscope is achieved by transferring the sample stage from the microscope focal point to the laser focal point.

Measurements.-For each ablation experiment the optimal measurement conditions, e.g. laser irradiance (pulse energy / (pulse width * crater area)) [ $\mathrm{TWcm}^{-2}$ ], total number of applied laser shots, laser pulse burst (sequences of laser pulses at a specific repetition rate), repetition rate and number of accumulated single mass spectra are defined according to the specific analyte.

In this contribution the pulse energy was varied from $0.8 \mu \mathrm{J}-$ $3.1 \mu \mathrm{J}$ for the ablation experiments and from $0.16 \mu \mathrm{J}-1.08 \mu \mathrm{J}$ for the desorption studies. The repetition rate was kept constant at $1 \mathrm{kHz}$ during all experiments.

\section{Results and Discussion}

$1 D$ depth profiling.-Galvanostatically deposited $\mathrm{Cu}$ films on blanket wafer coupons constitute an ideal model platform with welldefined uniformly-thick metal layers suitable to develop and test the depth profiling performance of the LMS system. Figure 3a shows a depth profile for Ta and Si signals for one such sample as a function of the number of applied laser pulses at $\sim 3.7 \mathrm{TWcm}^{-2}$ on a $10 \mu \mathrm{m}$-thick $\mathrm{Cu}$ electrodeposit prepared in the presence of the plating additives SPS and PAG (see entry 2 in Table I). ${ }^{53}$ The plot shows that about 6' 500 single pulses impinging the sample were needed to drill through the deposited $\mathrm{Cu}$ matrix and to reach the underlying $\mathrm{Ta} / \mathrm{TaN}$ buffer layer and the Si substrate. This strategy was repeated for a range of pulse energies to investigate the dependence of the mean ablation rate on the applied laser irradiance for distinct $\mathrm{Cu}$ samples deposited in the presence of several plating additives (Fig. 3b). ${ }^{53}$ The achieved mean vertical resolution was estimated by counting the number of laser pulses with a specific pulse energy necessary to completely penetrate the $\mathrm{Cu}$ layer (see Fig. 3c). The used $\mathrm{Si}(100)$ blanket wafer coupons (Hionix, provided by BASF) are terminated by a $100 \mathrm{~nm}$ thick PVD $\mathrm{Cu}$ seed-layer, a $25 \mathrm{~nm}$ thick Ta/TaN barrier layer and a $500 \mathrm{~nm}$ thick thermalized $\mathrm{SiO}_{2}$ dielectric layer.

These empirical studies demonstrated the suitability of LIMS to conduct 1D depth profiling with very high vertical resolution down to the nm scale and low ablation rates (fg to pg removal of material per single laser pulse) when applying laser irradiances just above the ablation threshold of $\mathrm{Cu}^{53}$ They further showed no effect of the 

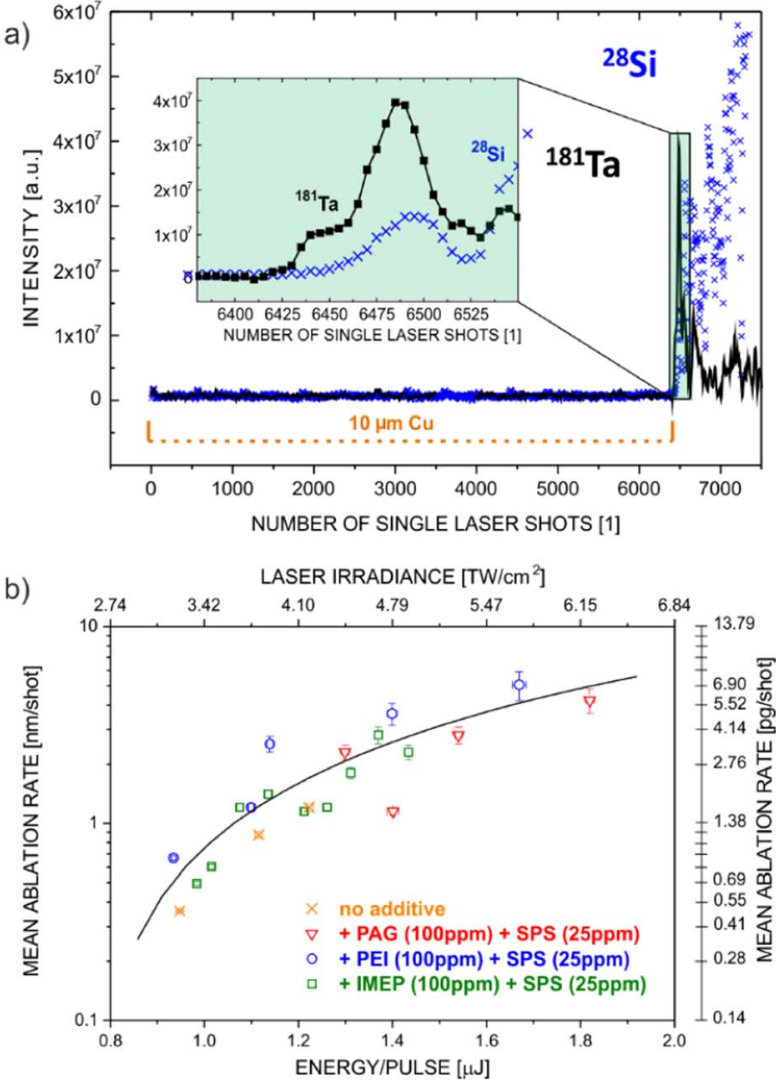

c)

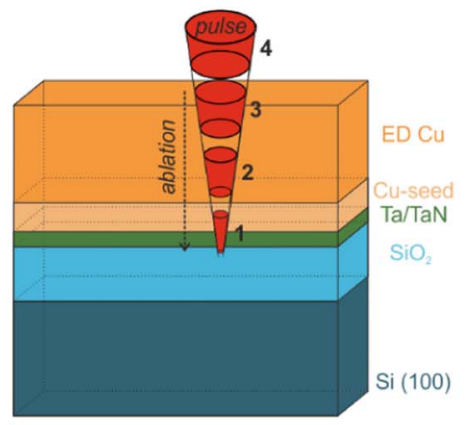

mean ablation rate:

\section{layer thickness $\{E D C u+C u$ seed + Ta/Ta $N\}$ Number of applied laser pulses}

Figure 3. a) Ta and $\mathrm{Si}$ signal evolution of the subjacent $25 \mathrm{~nm}$ thick Ta/TaN buffer layer and $\mathrm{Si}$ substrate through the $10 \mu \mathrm{m} \mathrm{Cu}$ electrodeposit. b) Evolution of the mean ablation rate with increasing pulse energy for four distinct $\mathrm{Cu}$ electrodeposits each bearing different plating additives. c) Schematic of the laser ablation experiment performed on the electrodeposited $\mathrm{Cu}$ film. It shows the number of laser pulses needed to penetrate the $\mathrm{Cu}$ layer down to the $\mathrm{SiO}_{2}$ substrate including thereby the ablation of the $\mathrm{Cu}$ seed and the $\mathrm{Ta} / \mathrm{TaN}$ buffer layers. Panel a) and b) reprinted (adapted) with permission from Ref. 53. Copyright (2015) American Chemical Society.

suppressing additives employed for the electrodeposition on the resulting ablation rate.

In a second stage of this $1 \mathrm{D}$ analytical development, we made use of a particular non-linear phenomenon of the SPS/IMEP-based plating additive package (see entry 4 in Table I) that enabled fabrication of multilayered $\mathrm{Cu}$ deposits as testbeds for advanced depth profiling investigations.

IMEP belongs to a new type of leveler additives, which exhibits a convoluted behavior of traditional type I (classical suppressor) and type II suppressors (classical levelers) in Damascene electroplating processes. ${ }^{12-14}$ While the working principle of classical type-II suppressors relies on specific mass transport limitations and consumptive inclusion in the deposited material, ${ }^{54-57}$ these hybrid-type additives exhibit a more selective activation that is not based on the transport and inclusion model. Contrary to conventional levelers this hybrid suppressor is, to some extent, already present inside the Damascene features before accomplishment of the bottom-up fill process. Linear sweep voltammetry (LSV) experiments of Cu electrodeposition in the presence of IMEP and SPS have demonstrated the occurrence of a partially hidden $\mathrm{N}$-shaped negative differential resistance $(\mathrm{N}-\mathrm{NDR})^{58}$ which is indicative for a delayed suppressor activation process superimposed on the primary $\mathrm{Cu}$ deposition. ${ }^{20,59} \mathrm{Hai}$ et al. have shown that at low overpotentials IMEP and SPS interact in an antagonistic way. After passing a certain transition regime the inactivation of IMEP by SPS at higher overpotentials is abrogated, indicating that only at sufficiently high overpotentials IMEP behaves as an effective leveler. In the course of the Damascene filling process the IMEP action is switched in a two step electrodeposition from a conventional type I suppressor behavior at the initial stage of the plating process to a leveler-type suppressor effect at the final stage of the superconformal filling. This new class of hybrid additives produces void-free interconnects with reduced metal overburden at their outermost surface and offers the opportunity to reduce the complexity of the plating formulation by using the same suppressor additive for the bottom up fill and the levelling processes. $^{20}$

Under specific galvanostatic deposition conditions (e.g. applied current density, additives concentration) this phenomenon is manifested by the appearance of characteristic periodic potential oscillations observable in the potential transients. Based on element analysis in combination with Super Conducting Quantum Interference Device (SQUID) measurements, Focused Ion Beam (FIB)-SEM and SIMS experiments, ${ }^{12-14}$ a $\mathrm{Cu}$ electrodeposition reaction mechanism was proposed, according to which the origin of such potential oscillations is a periodically repeating formation and degradation cycle of a suppressor ensemble at the solid-liquid interface superimposed to the reduction of $\mathrm{Cu}^{2+}$ ions under reactive conditions (see Fig. 2). According to present understanding, the suppressor ensemble consists of the IMEP polymer and multiple coordinated $\mathrm{Cu}(\mathrm{I})$-MPS ligands (where MPS is mercaptopropane sulfonic acid, a dissociation product of physiosorbed SPS molecule on $\mathrm{Cu}$ ) stabilized by intraand inter-chain interactions between the negatively charged sulfonate head group of the MPS molecules and the positively charged nitrogen atom of the imidazole rings. The accumulation of such electrostatic bonds transforms the pristine positively charged IMEP polymer into a neutral, hydrophobic polymer that precipitates at the solid-liquid interface. An eventual excess of free MPS, however, dissolves the active suppressor ensemble by cleaving the $\mathrm{O}-\mathrm{Cu}$ bond in the precipitate. To our understanding it is the concentration ratio between the free MPS and the $\mathrm{Cu}(\mathrm{I})$-MPS co-additive which regulates the structural breakdown and the restauration of the active suppressor ensemble. The latter tends to be embedded in the growing deposit when its dense network floating on top of it collapses. After post-deposition recrystallization at room temperature of the samples, these locally confined inclusions form delta-like periodically arranged grain boundaries perpendicular to the film growth direction.

To corroborate this mechanism, an elaborated chemical composition and molecular structure analysis of the acting suppressor ensemble forming such impurity layers was implemented. Figure $4 \mathrm{a}$ displays the potential transient of a $15.4 \mu \mathrm{m}$ thick test sample galvanostatically deposited at $j=-6 \mathrm{mAcm}^{-2}$ from plating bath 4 in Table I. ${ }^{60}$ The ordinate of the plot corresponds to the nominal measured potential and the abscissa to the charge transferred upon $\mathrm{Cu}$ electroplating. According to the proposed reaction mechanism, the dark-red sections of the transient curve are observed when the suppressor network is effectively floating on top of the surface, whereas the sharp potential transitions highlighted by orange appear when the suppressor network collapses and gets embedded inside the growing $\mathrm{Cu}$ deposit. The cross-sectional FIB-SEM analysis presented in Fig. 4b shows 


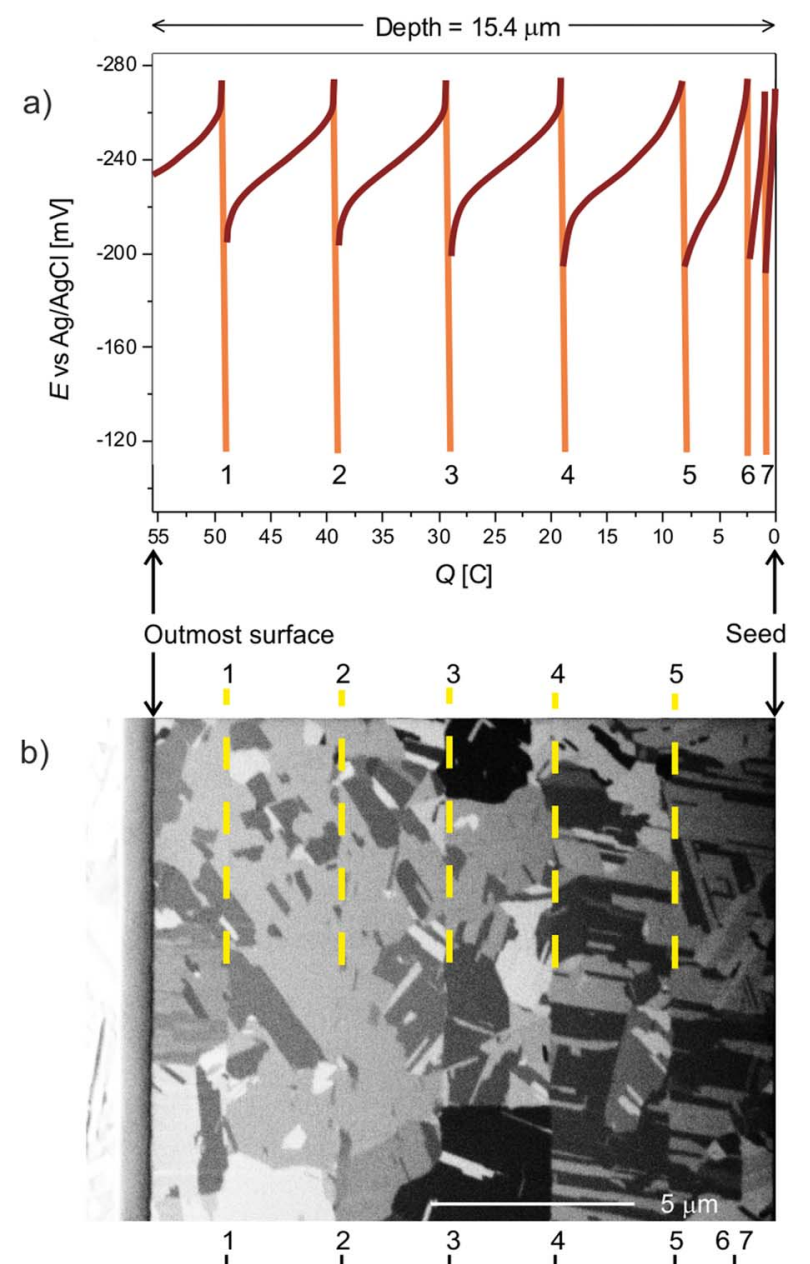

c)

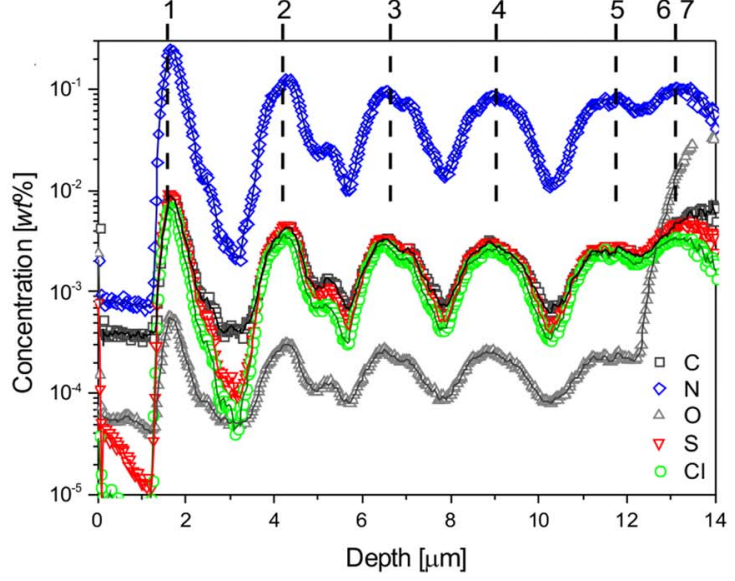

Figure 4. a) Potential-charge dependence of a galvanostatically deposited $\mathrm{Cu}$ sample at $j=-6 \mathrm{mAcm}^{-2}$ on a Cu-seeded $\mathrm{Si}(100)$ blanket wafer. b) Crosssectional FIB-SEM micrograph of an as-prepared sample. The dashed lines in the graph emphasize the horizontal grain boundaries, which spatially coincide with the potential oscillations in panel a. c) SIMS chemical depth profiles acquired in the negative mode showing the abundance fluctuation (wt\%) in depth of embedded contaminants, e.g. C, N, O, S, and Cl. Reprinted (adapted) with permission from Ref. 60. Copyright (2016) Elsevier.

sharp grain boundaries (emphasized by dashed yellow lines) that constrain well-recrystallized $\mathrm{Cu}$ domains and spatially coincide with the potential oscillations observed in panel a ${ }^{60}$ Standard depth profiling analyses by SIMS presented in Fig. 4c exhibit a periodic modulation of the $\mathrm{C}, \mathrm{N}, \mathrm{O}, \mathrm{S}$ and $\mathrm{Cl}$ signals within the $\mathrm{Cu}$ deposit whose peak center correlate with the grain boundaries revealed by the SEM images. ${ }^{60}$ However, in contrast to the manifest sharpness of these grain boundaries, the impurity intensities recorded by SIMS expand over a broader depth range and attenuate with progressive ion erosion. This is a SIMS instrument artifact and can be attributed to the surface roughening induced by the ion sputtering of the SIMS measurement, which smears and dilutes the embedded contaminants in the subjacent $\mathrm{Cu}$ layers (layer mixing). In addition to it, another drawback of the SIMS technique is its inherent strong matrix effects that prevent the quantification of the contaminants inside the electrodeposits and therefore prevent the stoichiometric analysis of the impurities.

To overcome these limitations of the SIMS technique we then conducted truly quantitative and sensitive LIMS depth profiling studies on these multilayered specimens. Figure 5 shows a chemical LIMS depth profile for the $\mathrm{C}, \mathrm{N}, \mathrm{O}, \mathrm{Cl}$ and $\mathrm{S}$ elements. ${ }^{53}$ The confined and controlled removal of material at very low ablation rates enables detection of abrupt periodic anti-correlated signals in the $\mathrm{Cu}$ and $\mathrm{C}, \mathrm{N}$, and $\mathrm{O}$ depth profiles that correspond to the sharp deltas shown by FIB micrographs similar to the ones in Fig. $4 \mathrm{~b}$ and thus further confirms the validity of the proposed reaction mechanism in Fig. 2. The same periodicity holds on only partially for the significantly lower abundant $\mathrm{S}$ and $\mathrm{Cl}$ impurities. In the marked up range of the depth profile in Fig. 5 it is clearly recognizable that in comparison to the SIMS measurement where a broad modulation of the impurities is observed, a specific elemental pattern for the individual layer becomes measurable characterizing the chemical composition of the contaminants at the location of their embedment.

The superior vertical resolution featured by the LIMS technique enabled more detailed understanding of the $\mathrm{Cu}$ deposits constitution as compared to the SIMS studies. ${ }^{61}$ The chemical profiles revealed that depending on the local contamination level at different depths, the impurities can be subdivided into three distinguishable regimes. ${ }^{60}$ The sample oxygen LIMS depth profile presented in Figure 6a exemplarily shows the first regime highlighted by blue of highest contribution of impurities (>10'000 ppm, atomic fraction (at\%)) and is ascribed to the periodic sharp horizontal grain boundaries formed upon collapse of the suppressor ensemble network. ${ }^{60}$ Opposed to this, the second regime highlighted by yellow is characterized by a very low contribution of contaminants $(<100 \mathrm{ppm} a t \%)$ and is assigned to well re-crystallized $\mathrm{Cu}$ domains located between the horizontal grain boundaries. This regime defines the background impurity level. The third regime highlighted by red shows an intermediate contamination level, and is characterized by the presence of satellite peaks that are at least twice as intense as the background signal but are about one decade less abundant than the intense peaks of the first regime. These satellite peaks are assigned to particular domains inside the $\mathrm{Cu}$ deposit, which do not comprise the sharp horizontal grain boundaries but feature a lower recrystallization degree of the material. Indeed, the positive correlation between impurity concentration and density of grain boundaries at any given depth suggests a preferential accumulation of contaminants at the boundaries of individual recrystallized $\mathrm{Cu}$ grains. ${ }^{60,61}$ Note, the plane of irradiation always comprises a certain number of grain boundaries and the analyzed total impurity concentration for the individual depth profiling regimes is referred to the sum of all boundaries striking the plane of laser irradiation. FIB crosssections I, II and III in Fig. 6b illustrate the correspondence between the extent of sampled grain boundaries and absolute organic impurity concentration proper of each described regime in panel a. The horizontal colored rectangles in each cross-section exemplifies the plane of ablation, which comprises different densities of grain boundaries depending on the vertical location inside the sample. The total impurity concentration of a single boundary is however not accessible with the current lateral resolution of the instrument $(\sim 15 \mu \mathrm{m})$. However, the quantitative nature of the LIMS technique enables a true determination of the sample chemical composition on the basis of a statistically enriched measurement campaign. As listed in Table II the contamination stoichiometry from the horizontal grain boundaries (regime I) differs from that of the randomly distributed boundaries inside the recrystallized $\mathrm{Cu}$ domains (regimes II and III). ${ }^{60}$ The fraction of $\mathrm{C}$ is substantially larger in the horizontal grain boundaries, indicating that they are mainly constituted of plating additives. The relative N/S 


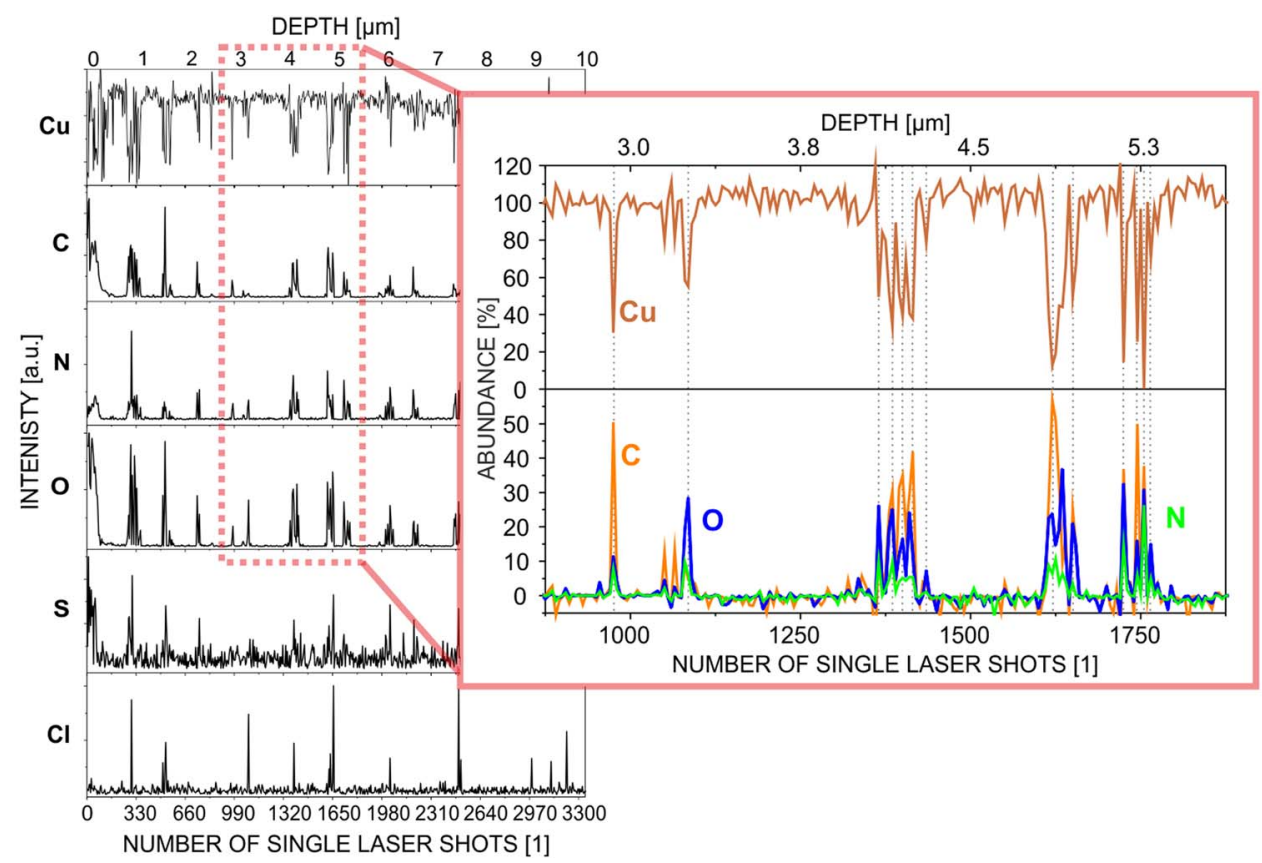

Figure 5. LIMS chemical depth profile of a $\mathrm{Cu}$ sample bearing multiple spatially confined contamination layers. The magnified region clearly shows the improved vertical resolution that enables a more detailed understanding of the impurity location as compared to SIMS results. Reprinted (adapted) with permission from Ref 53. Copyright (2015) American Chemical Society.

ratio in this region is $\sim 1.5$, referring further to the embedment of the suppressor ensemble with a non-stoichiometric contribution of leveler (IMEP) and antisuppressor (MPS), imposing that only a fraction of the leveler's OH-groups are effectively coordinated to MPS-Cu(I) ligands (Fig. 2). The regimes with randomly distributed boundaries (II and III) show, on the other hand, a dominant contribution of $\mathrm{O}$ pointing to a different impurity source, which is rather related to the embedment of either the supporting electrolyte (e.g. $\mathrm{SO}_{4}{ }^{2-}$ aq.) or the partially solvated sulfonate head groups from the SPS plating additive. In either case, the impurity incorporation originating from galvanostatic deposition carried out under conditions proper of these two regimes II and III is particularly low using the IMEP/SPS additive package. This low contamination level, which is also obtained when plating the $\mathrm{Cu}$ interconnects outside the N-NDR potential regime (as in the industrial process) is indeed the reason for the high post-deposition recrystallization degree and the formation of micrometer large $\mathrm{Cu}$ grains.

Molecular structure analysis.-In a more recent study going beyond the analysis of the pure element composition of the embedded impurities, a new matrix-free laser desorption experimental protocol was developed for the direct molecular structure recognition of the discussed suppressor ensemble. Structural information obtained from fragmentation patterns can disclose possible mechanism of action during the embedment process. Simplified testbeds were prepared to tune the appropriate measurement conditions that were applied in a further stage to the multilayered $\mathrm{Cu}$ deposits. ${ }^{62}$ They consisted of a dried drop-casted aqueous solution of pristine IMEP (see Fig. 7a), and a dried yellow precipitate of the suppressor ensemble (Fig. 7b). The latter was formed in a beaker-scale experiment by mixing IMEP and MPS in a certain concentration ratio (1 g/L IMEP and $24 \mathrm{~g} / \mathrm{L}$ MPS) in $8.04 \mathrm{~g} / \mathrm{L} \mathrm{CuSO}_{4}$ aqueous solution containing $10 \mathrm{~g} / \mathrm{L} \mathrm{H}_{2} \mathrm{SO}_{4}$ and $51.42 \mathrm{mg} / \mathrm{L} \mathrm{HCl}{ }^{13}$ The samples were subjected to milder laser irradiation for their molecular identification than in the case of the ablation studies (see Experimental section) ${ }^{62}$ Figure $7 \mathrm{c}$ displays the acquired mass spectra for the synthesized precipitate (top spectrum) and the pure IMEP polymer (bottom spectrum). Although upon application of gentle laser desorption conditions both testbeds were affected by severe fragmentation, clear molecular fingerprints related mainly to the IMEP backbone could be identified. At low $\mathrm{m} / \mathrm{z}$, values aliphatic side chains comprising the $\mathrm{OH}$-group are observed. The spectra regions highlighted by pink and violet emphasize the most abundant fragments which contain the imidazole ring. The most favored bondcleavages are indicated by the dotted lines on the lower right corner of the panel with the corresponding color code.

${ }^{63} \mathrm{Cu}^{+}$and ${ }^{65} \mathrm{Cu}^{+}$isotopes and additional species that could be assigned to thiolate-related fragments (e.g. $\mathrm{m} / z: 59\left[\mathrm{~S}-\mathrm{CH}_{2}-\mathrm{CH}\right]^{+}$and 72 $\left.\left[\mathrm{S}-\left(\mathrm{CH}_{2}\right)_{2}-\mathrm{C}\right]^{+}\right)$are only found in the suppressor ensemble spectrum. Finally, slight intensity deviation in the mass range $\mathrm{m} / \mathrm{z}=105-170$ further points to the superposition of isobaric molecular fragments that might be related to both, pristine IMEP or suppressor ensemble. Possible fragments are indicated by labels and are believed to involve the $\mathrm{O}-\mathrm{Cu}-\mathrm{S}$ bridge building block.

These findings support that under the applied experimental conditions, the LIMS-based matrix-free laser desorption analysis enables molecular recognition of the proposed suppressor ensemble at those locations of the layered testbeds where long range $\mathrm{Cu}$ recrystallization is inhibited. To analyze in-situ these impurity layers a two-step

Table II. LIMS impurity quantification of the multilayered $\mathrm{Cu}$ deposits prepared with the IMEP/SPS-based plating package. Reprinted with permission from Ref 60. Copyright (2016) Elsevier.

\begin{tabular}{cccccc} 
& Tot. impurity content [ppm, at\%] & $\mathrm{C}[\%]$ & $\mathrm{O}[\%]$ & $\mathrm{N}[\%]$ & $\mathrm{S}[\%]$ \\
\hline Regime I & 36800 & 47.00 & 33.00 & 10.90 & 7.70 \\
Regime II & 158 & 28.20 & 46.40 & 8.50 & 14.30 \\
Regime III & 1130 & 27.83 & 51.89 & 12.56 & 5.57
\end{tabular}




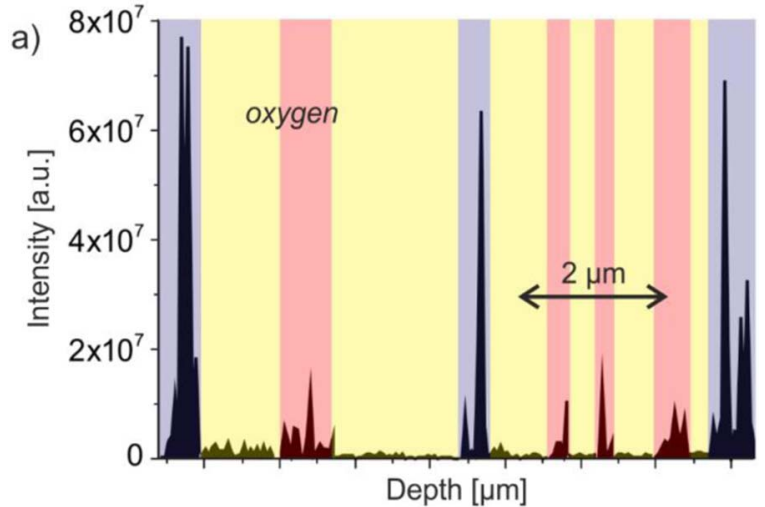

b)

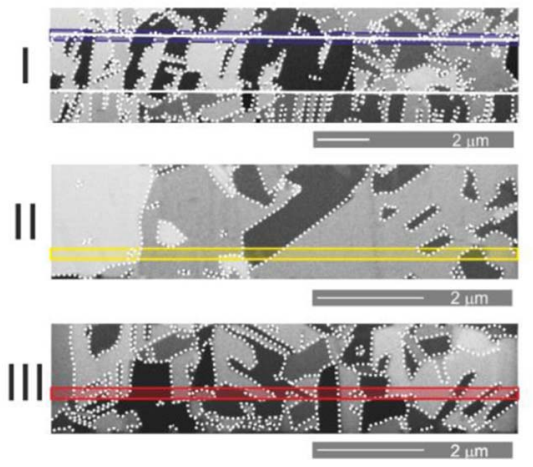

Figure 6. Sample oxygen depth profile of a multilayered $\mathrm{Cu}$ sample electrodeposited with the IMEP/SPS plating package subdivided into three distinct intensity regimes indicated in blue, yellow and red. Blue corresponds to those highly contaminated regimes originating from the sharp horizontal grain boundaries (highlighted area in cross-section I of panel b). Yellow sections represent the ablation planes striking well-recrystallized $\mathrm{Cu}$ domains with low amount of grain boundaries (highlighted area in cross-section II of panel b). The red sections enclose those analyzed areas encountered in $\mathrm{Cu}$ domains with a lower degree of recrystallization (highlighted area in cross-section III of panel b) but outside the well localized delta layers. Panel a) reprinted (adapted) with permission from Ref 60. Copyright (2016) Elsevier. a)
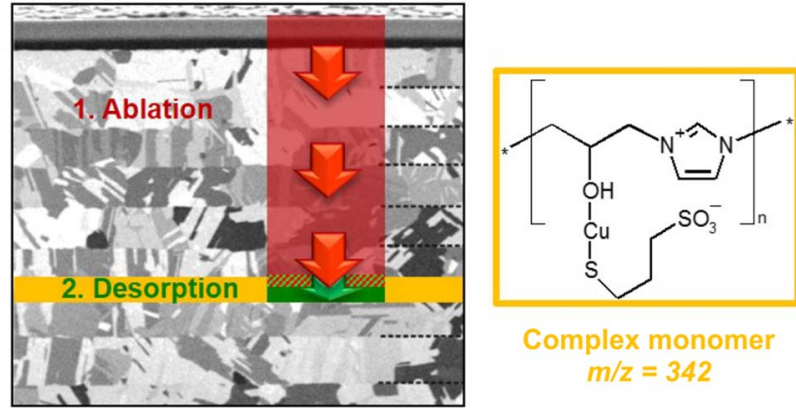

Complex monome $\mathrm{m} / \mathrm{z}=342$

b)

Single shot MS

Complex monomer

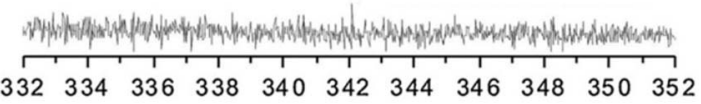

Complex monomer $-\mathrm{CH}_{2}$

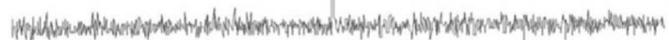

$\begin{array}{lllllllllll}318 & 320 & 322 & 324 & 326 & 328 & 330 & 332 & 334 & 336 & 338\end{array}$

$\mathrm{m} / \mathrm{z}$

Figure 8. a) Schematics of the two-step ablation matrix-free laser desorption approach on a multilayered Cu test sample. After reaching the layer of interest with the appropriate laser ablation conditions (indicated in red) the laser was resumed to gentle laser desorption irradiations (green arrow). b) Single pulse mass spectra showing the detection of the IMEP-Cu(I)-MPS monomer ensemble (see molecule structure in panel a) with and without the loss of a $\mathrm{CH}_{2}$ side chain. Panel b) reprinted (adapted) with permission from Ref 60. Copyright (2016) Elsevier.

measurement approach was applied. ${ }^{60}$ In a first stage the laser irradiance was set to $\sim 2.5 \mathrm{TWcm}^{-2}$ for strong ablation of the $\mathrm{Cu}$ matrix. The signal was thereby continuously monitored to follow online the signal evolution of the ablated material. Once an impurity layer similar to the one highlighted by the blue rectangle in Fig. 6b-I was reached, the laser irradiation was stopped and resumed after setting laser conditions just above the laser desorption threshold $\left(0.4-0.6 \mathrm{TWcm}^{-2}\right)$ of the organic material. Fig. 8a schematically illustrates these
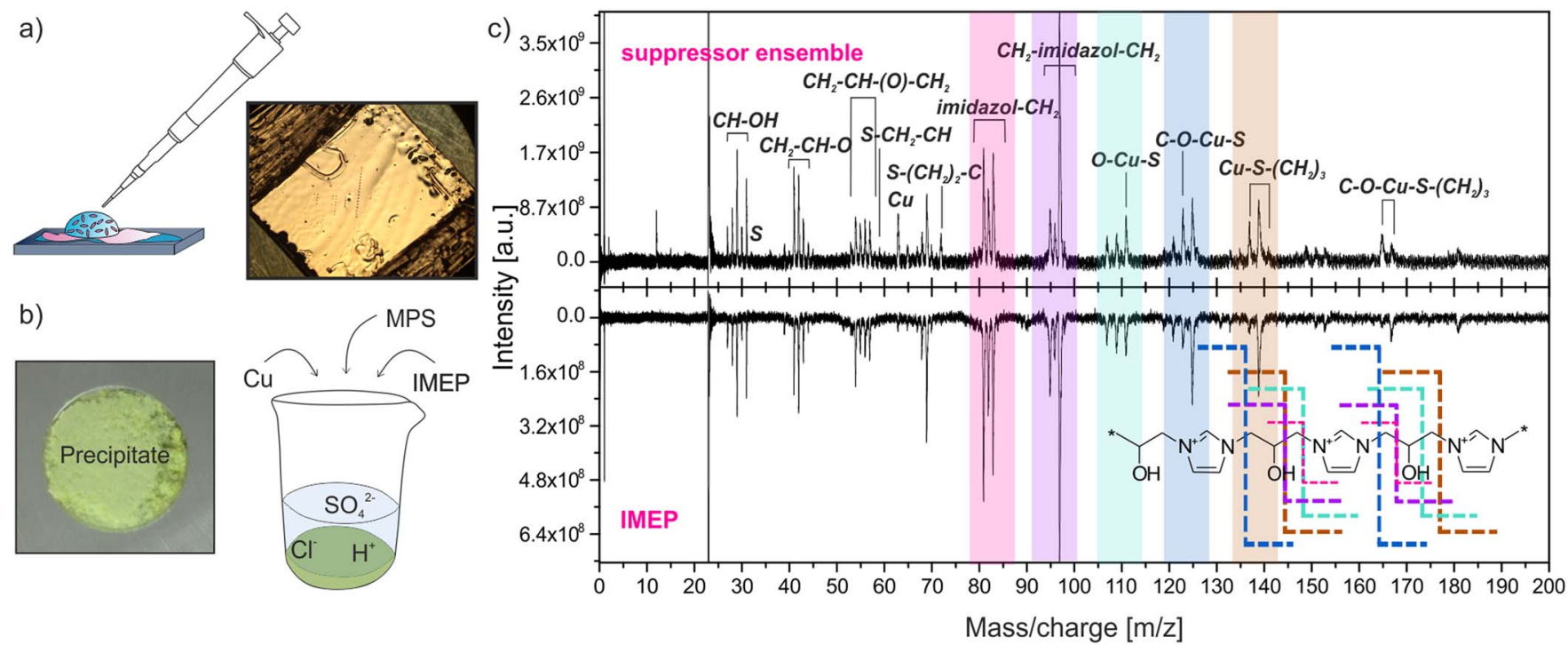

Figure 7. Preparation schemes of a) a drop-casted IMEP film on a Cu-seeded Si wafer and b) a pellet of the IMEP-Cu(I)-MPS suppressor ensemble synthesized in a beaker-scale experiment. c) Laser desorption mass spectra of the precipitated suppressor ensemble (top spectrum) and the pristine IMEP polymer (bottom spectrum). 
two-step approach. The red arrows represent the initial ablation process, where the ablated material is completely atomized. The underlying green arrow symbolizes the conduction of the desorption step that is applied on an impurity layer comprised within the yellow region. Note that the harsh irradiation from the former ablation step induces strong damage in the subjacent layers (shaded area) and leaves behind only a small fraction of intact organic network (green area). Nevertheless, the analysis of single pulse mass spectra allowed for the recognition of higher molecular masses that correspond to the mass of an IMEP-Cu(I)-MPS monomer (the repetition unit of the suppressor ensemble, see Fig. 8a). ${ }^{60}$ These findings coupled to the former element composition studies of the horizontal grain boundaries are consistent with the proposed reaction mechanism.

The capability of LIMS to not only perform spatial elementary chemical analysis but also molecular structure characterization without sample preparation enables it as an outstanding analytical tool that could shed light on topics relevant for the development of other additive-assisted electrochemical processes.

LIMS analysis of actual interconnect components.-The next step of our analytical development consisted in testing of the LMS performance on actual state-of-the-art $\mathrm{Cu}$ electronic interconnects, namely through-silicon-vias (TSV). ${ }^{4,63-65}$ These structures are large scale interconnects for the three-dimensional integration of multiple stacked transistor levels in cutting edge microprocessors. Their high aspect ratios constitute an important challenge for the electrochemical deposition of $\mathrm{Cu}$ and therefore new concepts for their successful additive-assisted electrochemical superfilling have been developed. ${ }^{65-68}$ A promising filling concept involves the formation of a suppressor concentration gradient along the TSV side-walls that sustains the suppressing effect on the time scale of minutes. However, an important drawback when applying such strong suppressing concepts is the local embedment of the employed polymers going along with the $\mathrm{Cu}$ deposition. As in the case of many other metal interconnects, the embedment of organic impurities inside the TSVs undermines their performance, reliability and lifetime. Therefore, improved suppressor additives that achieve the desired superconformal metal deposition with reduced incorporation into the electrodeposits are being pursued.

TSV structures deposited with one such new chemistry package were analyzed with the LMS instrument aiming at understanding the particular superfilling reaction mechanism of the employed plating chemistry and at quantifying its embedment in the $\mathrm{Cu}$ structure. This is, however, a more demanding task compared to the above addressed investigations due to the dimensions, geometry and multicomponent nature of TSVs. Additionally, the currently accessible laser spot $(\varnothing$ 10-20 $\mu \mathrm{m})$ exceeds the dimensions of the Cu-TSV opening $(\varnothing=5$ $\mu \mathrm{m})$ and therefore it also affects the surrounding $\mathrm{Si}(100)$ matrix upon irradiation on a single TSV. Particularly the distinct physical and chemical properties of the TSV major components (e.g. $\mathrm{Cu}$ and $\mathrm{Si}$ ) exhibit rather different laser-matter interactions (e.g. ablation threshold and rate $)^{69}$ which could induce an uneven removal of the irradiated TSV analyte. In fact, it is well known that uniform laser erosion of semiconductors requires harsher ablation conditions than metals. ${ }^{69,70}$ Therefore, optimal laser conditions had to be initially determined to promote a nonselective removal of the irradiated material above the $\mathrm{Si}$ ablation threshold that would at least reach the bottom of single TSV structures $(50 \mu \mathrm{m}$ deep). Thus dedicated LIMS ablation investigations were performed on the TSVs to determine optimal laser irradiances and number of applied laser pulses. ${ }^{71}$ An extensive ablation campaign consisting of a matrix with different number of laser pulses (burst number: $100,500,1$ '000 and 1'500) and applied energies $(2.4$ to $3.1 \mu \mathrm{J})$ was conducted. The depth and morphology of the laser-induced craters were determined by cross-sectioning them first through a combination of standard lithographic patterning techniques and anisotropic $\mathrm{Si}$ etching procedure (Bosch-process) and subsequently analyzing them by means of SEM imaging. ${ }^{69}$ Prior to the cross-sectioning step the sample surface was cleared from the $\mathrm{Cu}$-overburden allowing the etching of the material along the TSV features. ${ }^{71}$ The schematics of

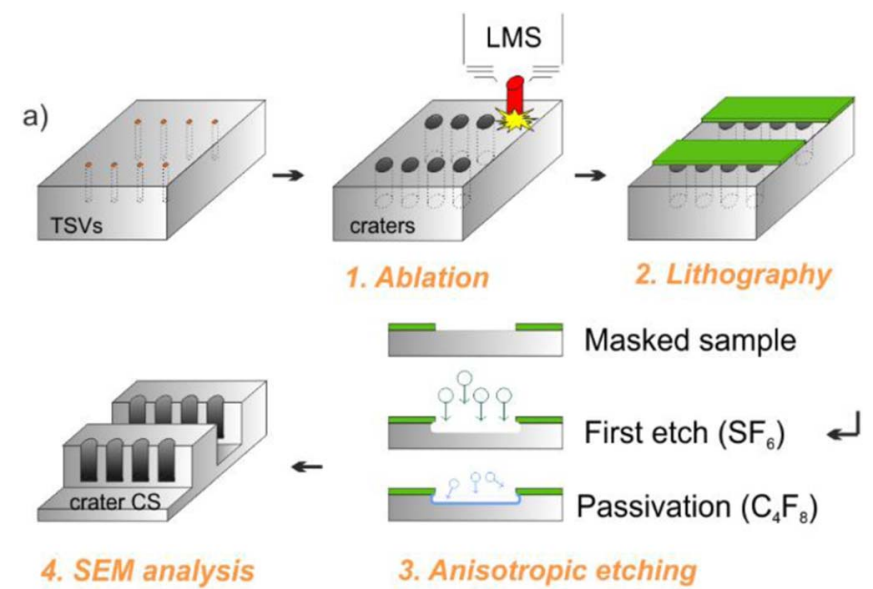

b)

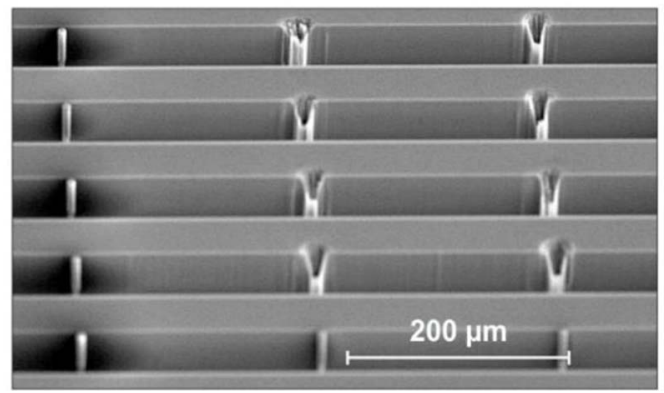

Figure 9. a) Schematics of the generation and cross-sectioning of LIMS craters on an array of TSV structures. The partial clearing of the craters is achieved by patterning lithographically a polymer mask on the sample covering the craters only half across their center. The unmasked region of the sample comprising the other half of the craters is then subjected to a sequence of alternating etching and passivation steps that promotes a directional removal of the sample in the laser irradiation direction. CS in panel (a) stands for cross-section. b) SEM overview of a cross-sectioned crater matrix formed on TSV structures. The matrix is comprised of (100-1'500) single laser shots and (2.4-3.1 $\mu \mathrm{J})$ applied pulse energies.

the cross-sectioning and a SEM overview of such an investigated sample are displayed in Fig. 9. The $\mathrm{Cu}$ channels of the non-ablated TSV structures were not affected by the etching step and are therefore protruding the cross-sectioned Si-matrix (bright features on the left side of Fig. 9b). The precise information about the crater depths allowed to estimate an absolute ablation rate for this dual $\mathrm{Cu}-\mathrm{Si}$ system that enabled the assignment of a depth scale in the obtained chemical depth profiles.

Next the spatial chemical analysis of actual single TSV features was carried out applying $10^{\prime} 000$ pulses at $\sim 2.5 \mu \mathrm{J}$ pulse energy on a statistically representative number of TSV structures. The ablated material from each single pulse generated a mass spectrum from which the composition was quantified and assigned to the specific sample depth. Besides the signals of the main matrix components, the results showed a non-linear decreasing trend of incorporated organic impurities with progressive depth (see Fig. 10a). ${ }^{71}$ Complementary Scanning Auger Microscopy (SAM) analysis of cross-sectioned TSV structures further allowed the exact local lateral assignment of the additive fingerprint (e.g. C) embedded in the TSV features (Fig. 10b). These experiments revealed a confined accumulation of $\mathrm{C}$ impurities at the feature side-walls and only a trace contribution of $\mathrm{C}$ inside the $\mathrm{Cu}$ via. ${ }^{71}$ These results are in full agreement with the initial working claim of an overgrown polymeric suppressor film located at the TSV side-walls. Furthermore, the particularly low $\mathrm{C}$ content inside the TSV is most likely the chemical origin for the long range recrystallization of $\mathrm{Cu}$ inside the structures. ${ }^{71}$ 


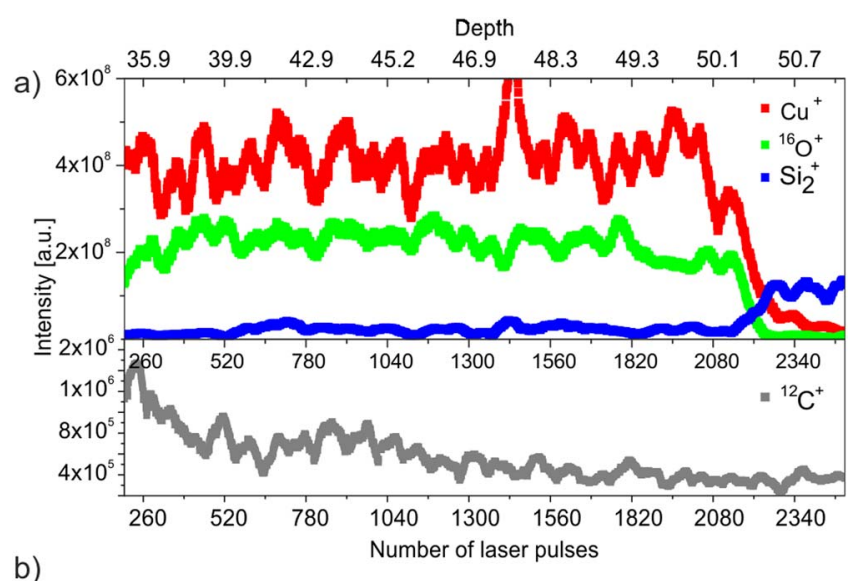

b)

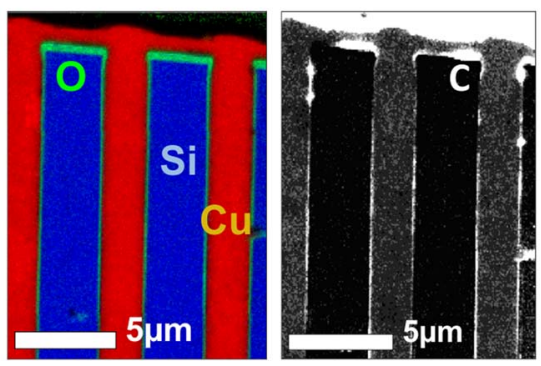

Figure 10. a) Sample LIMS depth profile showing $\mathrm{Cu}^{+}$(red), ${ }^{16} \mathrm{O}^{+}$(green), $\mathrm{Si}_{2}{ }^{+}$(blue) and ${ }^{12} \mathrm{C}^{+}$(gray) intensity signals as a function of applied number of laser pulses and calculated absolute depth. b) Scanning Auger Microscopy (SAM) of multiple TSV structures showing the presence of carbon impurities (second panel, white signal) preferentially embedded along the side-walls of the $\mathrm{Cu}$-lines. Reprinted (adapted) with permission from Ref 71. Copyright (2015) American Chemical Society.

\section{Conclusions}

The work presented in this review was motivated by the urge to better understand the electrochemical processes that determine the chemical composition of electrodeposited interconnects in state-ofthe-art integrated circuits (ICs). To address the chemical composition at the relevant spatial scales of these components applied in the microprocessor industry an elaborated spatial chemical analysis procedure was developed. Our investigations aimed in a first stage at quantitatively addressing the chemical composition of electrodeposited $\mathrm{Cu}$ film testbeds that utilized plating formulations typically applied in industrial interconnect manufacture by using the miniature fs-LIMS instrument designed and developed at the University of Bern. Comprehensive optimization of measurement conditions enabled $1 \mathrm{D}$ chemical depth profiling with unprecedented vertical resolution down to the $\mathrm{nm}$ scale. The analysis of organic impurities being potentially embedded in the deposits during the electrodeposition process was further investigated on test samples bearing multiple confined organic layers that were intentionally fabricated under non-linear deposition conditions in the presence of SPS and the hybrid IMEP suppressor. Combined LIMS, SIMS and FIB-SEM analyses enabled the identification of organic contaminants that preferentially accumulate at the grain boundaries of individual recrystallized $\mathrm{Cu}$ domains. These LIMS studies further enabled an accurate quantitative spatial chemical composition analysis of the layered samples which evidenced the depth-dependent occurrence of impurity embedment. Three distinct impurity embedment regimes were dominated either by O-related species indicating the embedment of supporting electrolyte or sulfonate head groups of the SPS molecule, or by C-containing species pointing to the embedment of the suppressor additive. The first case is assigned to wellrecrystallized domains, whereas the latter is exclusive to the confined delta-like impurity layers.
In a second phase, a novel LIMS-based matrix-free laser desorption approach was implemented, which goes beyond the pure elemental composition determination of solid matter and allowed for additional molecular structure understanding of the embedded contaminants. Molecular specific fragmentation patterns corroborated a previously suggested reaction mechanism describing the formation of an IMEP-Cu(I)-MPS suppressor ensemble acting on the growing metal deposit under specific deposition conditions (MPS = mercaptopropane sulfonic acid, the dissociation product of physisorbed SPS molecule). The determined N/S concentration ratio revealed a nonstoichiometric adduct formation between IMEP and MPS molecules. The found $\mathrm{S}$ deficit suggests that only a fraction of the $\mathrm{OH}$ linking groups of IMEP are effectively coordinated to the MPS-Cu(I) ligands.

Finally, the LIMS technique was applied for the first time on industrially metallized on-chip TSV systems. These investigations revealed the existence of carbon containing contaminants in the TSV structures that were preferentially incorporated on the upper part of the feature next to the sample surface. Complementary Scanning Auger Microscopy (SAM) analysis of the TSV cross-section identified a specific accumulation of the organic impurities at the side-walls of the electrodeposited $\mathrm{Cu}$ vias.

Ongoing work concerns the analysis of lead-free $\mathrm{Sn} / \mathrm{Ag}$ solder bumps that are deposited on $\mathrm{Cu}$ pillars and are applied to interconnect the circuit board to various devices e.g. integrated circuits (IC) and microelectrochemical systems (MEMS) ${ }^{72,73}$ The work addresses the investigation of possible organic contaminants and the appearance of intermetallic $\mathrm{Sn}-\mathrm{Ag}$ and $\mathrm{Sn}-\mathrm{Cu}$ phases that are formed along the feature.

\section{Acknowledgment}

This work is supported by the Swiss National Science Foundation. A. Riedo acknowledges the support from the European Union's Horizon 2020 research and innovation program under the Marie Skłodowska-Curie grant agreement No. 750353.

\section{ORCID}

Valentine Grimaudo (D) https://orcid.org/0000-0002-7010-5903

Pavel Moreno-García (DiD https://orcid.org/0000-0002-6827-787X

Andreas Riedo (10) https://orcid.org/0000-0001-9007-5791

Alena Cedeño López (D) https://orcid.org/0000-0002-4917-7355

Marek Tulej (D) https://orcid.org/0000-0001-9823-6510

Reto Wiesendanger (D) https://orcid.org/0000-0001-7127-0516

Peter Wurz (1D https://orcid.org/0000-0002-2603-1169

Peter Broekmann (D) https://orcid.org/0000-0002-6287-1042

\section{References}

1. P. C. Andricacos, C. Uzoh, J. O. Dukovic, J. Horkans, and H. Deligianni, IBM J. Res. Dev., 42(5), 567 (1998).

2. R. Akolkar, C.-C. Cheng, R. Chebiam, A. Fajardo, and V. Dubin, ECS Transactions, 2(6), 13 (2007).

3. V. M. Dubin, R. Akolkar, C. C. Cheng, R. Chebiam, A. Fajardo, and F. Gstrein, Electrochim. Acta, 52(8), 2891 (2007)

4. X. Gagnard and T. Mourier, Microelectron Eng, 87 (2010).

5. P. M. Vereecken, R. A. Binstead, H. Deligianni, and P. C. Andricacos, IBM J. Res. Dev., 49(1), 3 (2005).

6. T. P. Moffat and D. Josell, J. Electrochem. Soc., 159(4), D208 (2012).

7. T. P. Moffat and D. Josell, Israel J. Chem., 50(3), 312 (2010).

8. J. D. Reid and J. Zhou, ECS Trans., 2(6), 77 (2007).

9. O. Lühn, A. Radisic, P. M. Vereecken, C. Van Hoof, W. Ruythooren, and J. P. Celis, Electrochem. Solid-State Lett., 12(5), D39 (2009).

10. O. Lühn, C. Van Hoof, W. Ruythooren, and J. P. Celis, Electrochim. Acta, 54(9), 2504 (2009).

11. W.-P. Dow, C.-C. Li, Y.-C. Su, S.-P. Shen, C.-C. Huang, C. Lee, B. Hsu, and S. Hsu, Electrochim. Acta, 54(24), 5894 (2009).

12. N. T. M. Hai, K. W. Krämer, A. Fluegel, M. Arnold, D. Mayer, and P. Broekmann, Electrochim. Acta, 83(0), 367 (2012).

13. N. T. M. Hai, J. Odermatt, V. Grimaudo, K. W. Krämer, A. Fluegel, M. Arnold, D. Mayer, and P. Broekmann, J. Phys. Chem. C, 116(12), 6913 (2012).

14. N. T. M. Hai, D. Lechner, F. Stricker, J. Furrer, and P. Broekmann, ChemElectroChem, 2(5), 664 (2015). 
15. W. A. Rogers and J. E. Woehst, Vol. US 3,320,317. Dow Chemical Company, US 3.320.317, 1967.

16. F. Reniers and C. Tewell, J. Electron. Spectrosc. Relat. Phenom., 142(1), 1 (2005).

17. R. E. Galindo, R. Gago, D. Duday, and C. Palacio, Anal. Bioanal. Chem., 396(8), 2725 (2010)

18. T. J. Shaffner, Proceedings of the IEEE, 88(9), 1416 (2000)

19. J. C. Vickerman and N. Winograd, Int. J. Mass spectrom., 377568 (2015).

20. N. T. M. Hai and P. Broekmann, ChemElectroChem, 2(5), 664 (2015).

21. S. J. M. Van Malderen, J. T. van Elteren, and F. Vanhaecke, Anal. Chem., 87(12), 6125 (2015).

22. J. Koch and D. Günther, Appl. Spectrosc., 65(5), 155A (2011).

23. A. Gutiérrez-González, C. González-Gago, J. Pisonero, N. Tibbetts, A. Menéndez, M. Vélez, and N. Bordel, J. Anal. At. Spectrom., 30(1), 191 (2015).

24. E. H. Evans, J. Pisonero, Clare M. M. Smith, and R. N. Taylor, J. Anal. At. Spectrom., 33(5), 684 (2018)

25. V. Zorba, X. Mao, and R. E. Russo, Spectrochim. Acta B, 11337 (2015).

26. B. Fernández, R. Pereiro, and A. Sanz-Medel, Anal. Chim. Acta, 679(1-2), 7 (2010).

27. F. Hillenkamp, E. Unsöld, R. Kaufmann, and R. Nitsche, Applied physics, 8(4), 341 (1975)

28. U. Rohner, J. A. Whitby, and P. Wurz, Meas. Sci. Technol., 14(12), 2159 (2003).

29. M. Tulej, M. Iakovleva, I. Leya, and P. Wurz, Anal. Bioanal.Chem., 399(6), 2185 (2011).

30. A. Riedo, M. Neuland, S. Meyer, M. Tulej, and P. Wurz, J. Anal. At. Spectrom., 28(8), 1256 (2013)

31. M. B. Neuland, S. Meyer, K. Mezger, A. Riedo, M. Tulej, and P. Wurz, Planet. Space Sci., $101196(2014)$

32. M. Tulej, A. Neubeck, M. Ivarsson, A. Riedo, M. B. Neuland, S. Meyer, and P. Wurz Astrobiology, 15(8), 669 (2015).

33. W. B. Brinckerhoff, G. G. Managadze, R. W. McEntire, A. F. Cheng, and W. J. Green, Rev. Sci. Instrum., 71(2), 536 (2000).

34. G. G. Managadze, P. Wurz, R. Z. Sagdeev, A. E. Chumikov, M. Tuley, M. Yakovleva N. G. Managadze, and A. L. Bondarenko, Solar System Research, 44(5), 376 (2010).

35. R. Huang, Q. Yu, L. Li, Y. Lin, W. Hang, J. He, and B. Huang, Mass Spectrom. Rev., 30(6), 1256 (2011).

36. J. F. Moore, S. Milasinovic, R. J. Gordon, and L. Hanley, Rev. Sci. Instrum., 83(9), 093702 (2012)

37. Y. Cui, C. Bhardwaj, S. Milasinovic, R. P. Carlson, R. J. Gordon, and L. Hanley, ACS Applied Materials \& Interfaces, 5(19), 9269 (2013).

38. L. Giron-Monzon, L. Manelyte, R. Ahrends, D. Kirsch, B. Spengler, and P. Friedhoff, J. Biol. Chem., 279(47), 49338 (2004).

39. J. S. Becker, Inorganic Mass Spectrometry: Principles and Applications, John Wiley \& Sons Ltd, The Atrium, Southern Gate, Chichester, West Sussex PO198SQ, England (2007).

40. R. Hergenroder, O. Samek, and V. Hommes, Mass Spectrom. Rev., 25(4), 551 (2006).

41. R. E. Russo, X. Mao, J. J. Gonzalez, and J. Yoo, Spectroscopy (Duluth, MN, United States), 28(1), 24 (2013). CODEN: SPECET; ISSN: 0887-6703.

42. D. J. Hwang, H. Jeon, C. P. Grigoropoulos, J. Yoo, and R. E. Russo, Appl. Phys. Lett. 91(25), 251118 (2007).

43. A. Riedo, A. Bieler, M. Neuland, M. Tulej, and P. Wurz, J. Mass Spectrom., 48(1), 1 (2013).
44. A. Riedo, S. Meyer, B. Heredia, M. B. Neuland, A. Bieler, M. Tulej, I. Leya, M. Iakovleva, K. Mezger, and P. Wurz, Planet. Space Sci., 871 (2013).

45. T. P. Moffat, D. Wheeler, and D. Josell, J. Electrochem. Soc., 151(4), C262 (2004)

46. M. Tulej, A. Riedo, M. Iakovleva, and P. Wurz, International Journal of Spectroscopy, 2012 (2012).

47. A. Bieler, K. Altwegg, L. Hofer, A. Jäckel, A. Riedo, T. Sémon, P. Wahlström, and P. Wurz, J. Mass Spectrom., 46(11), 1143 (2011)

48. M. Tulej, R. Wiesendanger, A. Riedo, G. Knopp, and P. Wurz, J. Anal. At. Spectrom., 33(8), 1292 (2018)

49. Y. Lin, Q. Yu, W. Hang, and B. Huang, Spectrochimica Acta Part B: Atomic Spectroscopy, 65(11), 871 (2010).

50. A. Riedo, M. Tulej, U. Rohner, and P. Wurz, Rev. Sci. Instrum., 88(4), 045114 (2017).

51. S. Meyer, A. Riedo, M. B. Neuland, M. Tulej, and P. Wurz, J. Mass Spectrom., 52(9), 580 (2017)

52. R. Wiesendanger, D. Wacey, M. Tulej, A. Neubeck, M. Ivarsson, V. Grimaudo, P. Moreno-García, A. Cedeño-López, A. Riedo, and P. Wurz, Astrobiology, 18(8), 1071 (2018).

53. V. Grimaudo, P. Moreno-García, A. Riedo, M. B. Neuland, M. Tulej, P. Broekmann, and P. Wurz, Anal. Chem., 87(4), 2037 (2015).

54. D. Roha and U. Landau, J. Electrochem. Soc., 137(3), 824 (1990).

55. C. Madore, M. Matlosz, and D. Landolt, J. Electrochem. Soc., 143(12), 3927 (1996).

56. C. Madore and D. Landolt, J. Electrochem. Soc., 143(12), 3936 (1996).

57. J. J. Kelly, C. Tian, and A. C. West, J. Electrochem. Soc., 146(7), 2540 (1999)

58. K. Krischer, N. Mazouz, and P. Grauel, Angew. Chem. Int. Ed., 40(5), 851 (2001).

59. P. Strasser, M. Eiswirth, and M. T. M. Koper, J. Electroanal. Chem., 478(1-2), 50 (1999).

60. P. Moreno-García, V. Grimaudo, A. Riedo, M. Tulej, M. B. Neuland, P. Wurz, and P. Broekmann, Electrochim. Acta, 199394 (2016).

61. A. Riedo, V. Grimaudo, P. Moreno-Garcia, M. B. Neuland, M. Tulej, P. Wurz, and P. Broekmann, J. Anal. At. Spectrom. , 30(12), 2371 (2015).

62. P. Moreno-García, V. Grimaudo, A. Riedo, M. Tulej, P. Wurz, and P. Broekmann, Rapid Commun. Mass Spectrom., 30(8), 1031 (2016).

63. P. Garrou, P. Ramm, and M. Koyanagi, in Handbook of 3 D Integration, p. 1, WileyVCH Verlag GmbH \& Co. KGaA, (2014).

64. J. P. Gambino, S. A. Adderly, and J. U. Knickerbocker, Microelectron. Eng., 135(0), $73(2015)$.

65. W.-W. Shen and K.-N. Chen, Nanoscale Research Letters, 12(1), 56 (2017).

66. V. H. Hoang and K. Kondo, Electrochim. Acta, 212 (Supplement C), 270 (2016).

67. R. Akolkar, ECS Electrochemistry Letters, 2(2), D5 (2013).

68. A. Radisic, O. Lühn, J. Vaes, S. Armini, Z. El-Mekki, D. Radisic, W. Ruythooren, and P. M. Vereecken, ECS Transactions, 25(38), 119 (2010).

69. V. Grimaudo, P. Moreno-García, A. C. López, A. Riedo, R. Wiesendanger, M. Tulej, C. Gruber, E. Lörtscher, P. Wurz, and P. Broekmann, Anal. Chem., 90(4), 2692 (2018).

70. R. Baerbel, S. I. Dmitriy, E. G. Martin, and I. A. Sergei, J. Phys. D: Appl. Phys., 50(19), 193001 (2017)

71. V. Grimaudo, P. Moreno-García, A. Cedeño López, A. Riedo, R. Wiesendanger, M. Tulej, C. Gruber, E. Lörtscher, P. Wurz, and P. Broekmann, Anal. Chem., 90(8), 5179 (2018)

72. S. W. Jung, J. P. Jung, and Y. Zhou, IEEE Transactions on Electronics Packaging Manufacturing, 29(1), 10 (2006).

73. Y. Liu, J. Wang, L. Yin, P. Kondos, C. Parks, P. Borgesen, D. W. Henderson, E. J. Cotts, and N. Dimitrov, J. Appl. Electrochem., 38(12), 1695 (2008) 